\title{
Network Analysis of Massively Collaborative Creation of Multimedia Contents
}

\author{
Case Study of Hatsune Miku videos on Nico Nico Douga
}

\author{
Masahiro Hamasaki \\ National Institute of AIST \\ / JST, CREST \\ 1-1-1 Umezono, Tsukuba \\ Ibaraki, Japan \\ hamasaki@ni.aist.go.jp
}

\author{
Hideaki Takeda \\ National Institute of \\ Informatics (NII) \\ 2-1-2 Hitotsubashi, Chiyoda \\ Tokyo, Japan \\ takeda@nii.ac.jp
}

\author{
Takuichi Nishimura \\ National Institute of AIST \\ I JST, CREST \\ 1-1-1 Umezono, Tsukuba \\ Ibaraki, Japan \\ taku_ni4@ni.aist.go.jp
}

\begin{abstract}
The World Wide Web supports new styles of creative activities. For this study, we investigate massively collaborative creation via the Web, by which numerous people gather to evolve their works collaboratively. Nico Nico Douga is a video sharing website, where many videos are created collaboratively. We specifically examine Hatsune Miku, a version of singing synthesizer application software that has inspired not only song creation but also songwriting, illustration, and video editing. As described herein, different types of creators interact to create new contents though their social network. Using tags, we classified videos and creators on Nico Nico Douga automatically into four basic categories. Thereby, we produced a social network from relationships among videos and creators by analyzing videos' descriptions. The social network reveals interesting features. Different categories of creators serve different roles in evolving the network, e.g., songwriters gather more links than other categories, implying that they are triggers to network evolution. We also extracted communities from the network and observed different community structures. One is a centralized network in which a single songwriter is central and others are peripheral. The other is a messier network, in which some illustrators are central, but the centrality is weak.
\end{abstract}

\section{Categories and Subject Descriptors}

H.4 [Information Systems Applications]: Miscellaneous

\section{General Terms}

Human Factors

\section{Keywords}

Massively collaborative creation, Community analysis, Video sharing

Permission to make digital or hard copies of all or part of this work for personal or classroom use is granted without fee provided that copies are not made or distributed for profit or commercial advantage and that copies bear this notice and the full citation on the first page. To copy otherwise, to republish, to post on servers or to redistribute to lists, requires prior specific permission and/or a fee.

uxTV'08, October 22-24, 2008, Silicon Valley, California, USA.

Copyright 2008 ACM 978-1-60558-100-2/08/10 ...\$5.00.

\section{INTRODUCTION}

The Web has become a common platform for communication in our society. As a platform for communication, the Web presents various advantages over older ones. For example, it erases the sense of distance because people at any place can mutually communicate. It enables massive interactive collaboration through large-scale BBS and social tagging, which is impossible in real-world communication channels. It also enables large-scale information sharing not only of texts but also of various multimedia contents such as videos. YouTube is a typical example.

Such new features of communication have fostered a new style of creative activity. Of these, a very interesting feature is massively collaborative creation of digital content. Now people create new content by communicating or collaborating with others via the Web. One new feature of collaboration is the style of participation. Because vastly numerous people are involved, they often do not know each other. Numerous evolved or inspired versions of the video are created if a video explores a new idea or catches on. Another difference is the digital re-use of content. Some components of a work are re-used in evolved or inspired versions, e.g., an image or sound in a video can be re-used in a new version of the video. Consequently, digital work can be developed among people via the Web.

We are interested in the development of such digital works, particularly in the process as it unfolds in a video sharing website. We selected a video sharing website called Nico Nico Douga ${ }^{1}$, where intense collaborative video creation is occurring. We specifically address Hatsune Miku ${ }^{2}$, which is a version of singing synthesizer application software that has inspired many people to produce various music, picture, and video compositions. The Hatsune Miku phenomenon is interesting because different creators such as songwriters, song creators, illustrators, and CG creators interact in projects.

We adopted a method of social networking analysis as a basic method to investigate the phenomenon. Applying social network analysis method to web contents has yielded important results for explicating the structure of interaction among people (see [4]). In our case, we investigate how different types of creators interact to create new content through their social network.

The sections of this paper are organized as follows. We

\footnotetext{
${ }^{1}$ http://www.nicovideo.jp/

${ }^{2}$ http://www.crypton.co.jp/mp/pages/prod/vocaloid/cv01.jsp
} 
briefly describe related work in Section 2 and the target of our analysis-Nico Nico Douga and Hatsune Miku - in Section 3. Subsequently, we explain our data extracted from the website and the preliminary process for the analysis described in Section 4. We present the results of our analysis in Section 5 and summarize this report in Section 6 .

\section{RELATED WORK}

The analysis of citation relations is a traditional approach to investigate the development of scientific activities [6]. Much analysis and visualization of citation networks has been done recently because large volumes of bibliographic information are now available [1]. However, few reports among scientific papers describe creative activities. Cheng et al. [2] investigated data of video posts and networks of users and videos on YouTube. Nevertheless, their analysis of large amounts of data is interesting, but the analysis is superficial: it does not address the semantics of video creation. Halvey and Keane [3] made a similar analysis of YouTube.

\section{THE TARGET}

\subsection{Nico Nico Douga}

Nico Nico Douga is the most popular video sharing website in Japan. Started in December 2006, it now has more than 7.9 million users (1 July, 2008) and has published more than 0.8 million videos (estimated by the authors). The basic service closely resembles that of YouTube, but it has some unique functions and has gathered many users rapidly. The most interesting and unique function is the direct overlaying of comments on videos. A user can add comments about a specific playback time at a specific position in the video (see Fig. 1), which gives people a sense of sharing the viewing experience virtually. Furthermore, the creator can instantly know which specific moment or specific scene is appreciated by a viewer. It inspires the original creator or other creators to produce new videos to match such effects.

The birth and development of Hatsune Miku suggests a new direction to MAD movies. In Hatsune Miku videos, extracts are not taken from commercial programs; instead, the community creates videos.

\subsection{Hatsune Miku and Its Boom}

Hatsune Miku is a version of singing synthesizer application software. The singing synthesizer developed by Yamaha Corp. enables users to synthesize songs that include singing by inputting lyrics and melody on computers just like computer music. Hatsune Miku is a version of singing synthesizer applications called Vocaloid2; its uniqueness is that it adopted recorded vocals of a famous anime actress. Initially most of the created songs were existing songs, but original songs soon appeared.

At the same time, a mascot image of Hatsune Miku has caught on. There is only a single illustration of Hatsune Miku: it is printed on the software package. People draw and post different illustrations of Hatsune Miku. Then people started to create videos, such as promotion videos for musicians, with such original songs and drawings. Some people even create 3D models of Hatsune Miku and create $3 \mathrm{D}$ animation videos.

The mixture of different creation types is very interesting. Hatsune Miku gathered different types of creators: songwriters from the computer music field, illustrators who come

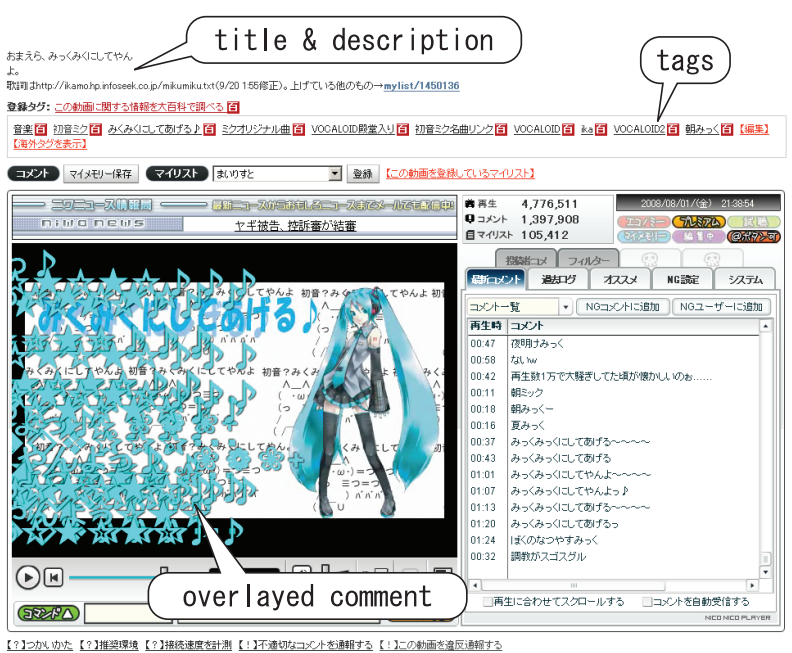

Figure 1: Screenshot of Nico Nico Douga. This video is one of the most popular videos related to Hatsune Miku.

from 'doujinshi' - self-published manga culture - and even CG creators. Most are amateurs, but some are professionals. They are stimulated by the work of others.

We categorize creation related to Hatsune Miku before the analysis.

- (a) Songwriting: Amateur songwriters are eager to promote their songs, but it takes time and money to produce promotion tapes with professional singers. Vocaloid solves this problem. Now they can produce sound with vocalizations as computer music. It inspires amateur songwriters to publish their original songs with Hatsune Miku.

- (b) Song creation: It is not easy to make Hatsune Miku sing songs naturally. Certain techniques are necessary to tune Hatsune Miku, but it is fun to tune the software to create nice singing songs. They vie with each other to create them.

- (c) Illustration: The image of Hatsune Miku is a typical anime character and attracts anime fans. They are used to drawing their favorite characters by themselves. They produce many different scenes and facial expressions. Those with more expertise produce animation. They often use illustrations drawn by others as material for producing their anime. Some produce $2 \mathrm{D}$ animation and others $3 \mathrm{D}$ animation with $3 \mathrm{D}$ CG tools.

- (d) Editing: There are so many Hatsune Miku videos that some people collect them and produce summary videos, with ranking programs of Hatsune Miku videos.

\section{DATA COLLECTION}

In all, 36,709 videos had the tag 'Hatsune Miku' on Nico Nico Douga (31 May 2008). From among them, we selected 7,138 videos that had been viewed more than 3,000 times as data for this study. We crawled their metadata during 1-5 June 2008. The metadata include view times, uploaded date, uploader name, tags, and a description. The most popular 


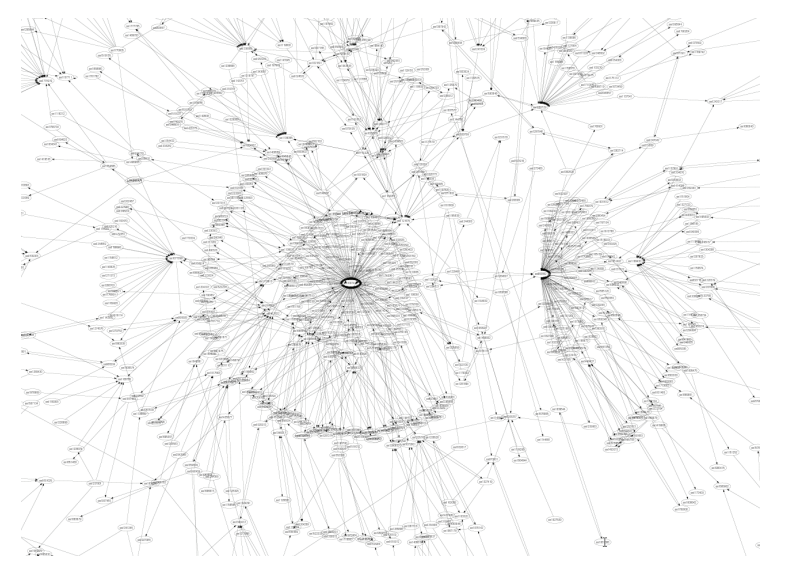

Figure 2: Reference Network

Table 1: The top five videos which have many links.

\begin{tabular}{|c|c|c|c|}
\hline Rank & $\begin{array}{c}\text { \# of } \\
\text { in-links }\end{array}$ & Upload date & $\begin{array}{c}\text { Category } \\
\text { of Creation }\end{array}$ \\
\hline 1 & 237 & $2007 / 09 / 20$ & Songwriting \\
\hline 2 & 124 & $2007 / 09 / 04$ & $\begin{array}{l}\text { Song creation } \\
\& \text { Illustration }\end{array}$ \\
\hline 3 & 93 & $2008 / 02 / 34$ & Illustration \\
\hline 4 & 74 & $2007 / 12 / 07$ & Songwriting \\
\hline 5 & 55 & $2007 / 09 / 13$ & Songwriting \\
\hline
\end{tabular}

video was viewed 4,425,208 times. The average number of views of the collected videos was 24,028 . These 7,138 videos were uploaded by 2,911 unique contributors ${ }^{3}$.

\section{THE RESULT OF THE ANALYSIS}

\subsection{Reference Network}

Each video has a title and a description written by the creator. The description often includes hyperlinks to other videos that can reflect details of the video's creation. It is customary on Nico Nico Douga that a creator cites other videos if a sound, image, or any part of another video is used. By tracing these hyperlinks, we generated a reference network of videos. Among the collected videos, 4,585 videos include hyperlinks in the description; we obtained 12,507 links among videos. Figure 2 shows a part of that network.

Two videos gathered many links around the center of the network. The left video published the most popular original song for Hatsune Miku. The right one introduced a character called "Hachune Miku" (an infantilized version of the Hatsune Miku mascot) with a leek. This video contributed to making Hatsune Miku popular.

We infer a relation between the creators of videos $A$ and $B$ when video $A$ has links to video $B$. In this way, we generated a network among creators. This network has 2,164 nodes (creators) and 4,368 links (relationships among creators). As described in this paper, we regard this network as a social network of creators.

\subsection{Category of Creation Activity}

In section 3.2, we classified creative activities related to

\footnotetext{
${ }^{3}$ On Nico Nico Douga, only the uploader is named. That person is not necessarily the creator of the video. However, for this study, we regard an uploader as the video creator.
}

Table 2: Categories of creation for creators: $W$ signifies Songwriting, $C$ means Songcreation, and $I$ represents Illustration.

\begin{tabular}{c|cc}
\hline Category & Creators & Sum of \#in-links \\
\hline W & 284 & 590 \\
I & 529 & 487 \\
C & 642 & 267 \\
W\&C & 75 & 351 \\
W\&I & 24 & 21 \\
C\&I & 44 & 176 \\
C\&W\&I & 17 & 36 \\
unknown & 1,296 & 349 \\
\hline total & 2,911 & 2,277 \\
\hline
\end{tabular}

Hatsune Miku into four categories: Songwriting, Song creation, Illustration, and Editing. In this section, we present the analysis of data based on these categories. Editing is more of a meta-creation activity than the others are. For that reason, we used the former three categories.

We classified videos and creators into creation categories automatically using tags for videos. Tags on Nico Nico Douga include not only review or content categories like 'cool', 'great', and 'music', but also kinds of creation: 'Original song', 'Make It Sing', and 'Make It Dance'. We provide a set of tags that are important for specific creation categories. For example, if a video has the tag "Miku Original Song", then we classify it as "Songwriting". If a video has the tag "3D Hatsune Miku Project", then we classify it into "Illustration". A video is classifiable into two or three categories if it has tags for these categories. We also determine the creator's category by aggregating that person's works. A single creator is classifiable into plural categories if the videos are classified into more than a single category ${ }^{4}$.

Among 2,911 creators, we obtained eight categories: three basic types, four types by combining basic categories, and an unknown category. Furthermore, we manually excluded 179 Editing videos.

Table 3 presents the number of creators and the number of in-links per creator by these categories. It might be readily apparent that the Songwriting and Song Creation category attracts many links, whereas Illustration does not do so. That fact suggests that Songwriting and Song Creation triggers creative activities. On the other hand, Illustration acquires many creators. Illustration activity is good at attracting collaborators.

Figure 3 portrays the relationship among creation categories. In this network, the node size represents the number of creators of each category; the arc width shows the number of relationships among creators. We draw an arc among categories when the relationships number more than 30. By following the links inversely, we can observe how the creative activity is diffused among different types of creators. Songwriters and Song Creators are the major source of the diffusion. Then Song Creators (only) and Illustrators followed the diffusion. Illustrators were followed by themselves, which indicates that they diffuse Hatsune Miku in their community.

\footnotetext{
${ }^{4}$ Some videos cannot be categorized correctly using this method. We checked some top-ranking videos and creators and corrected them manually.
} 


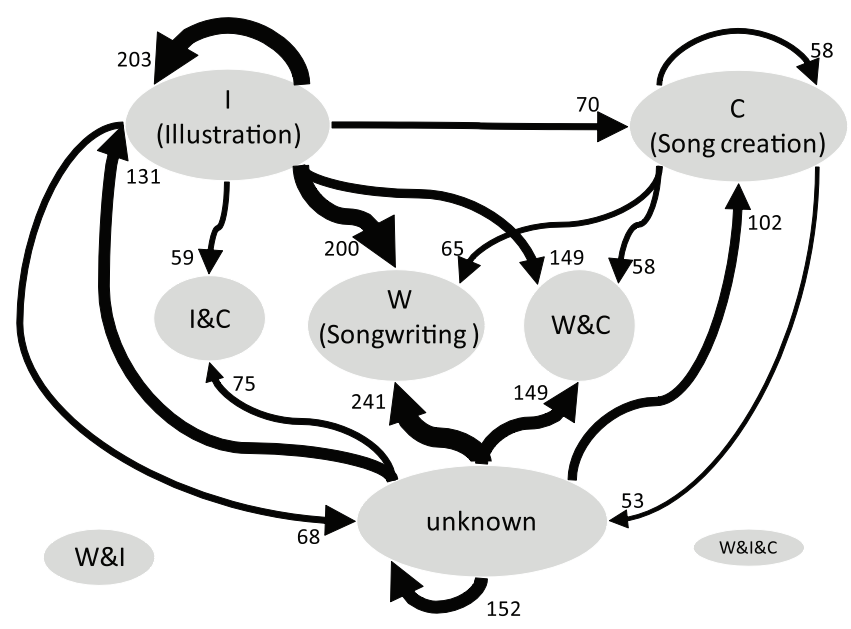

Figure 3: Relationship among categories of creation.
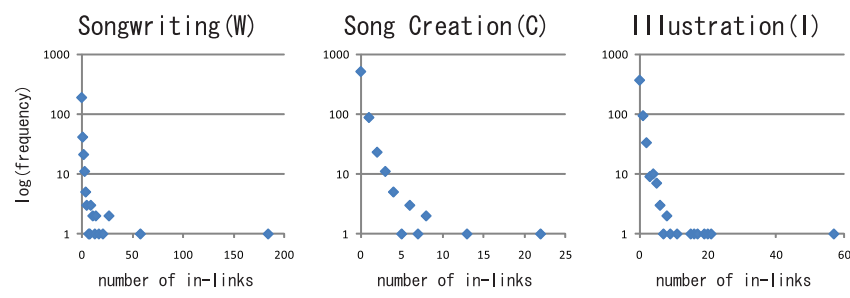

Figure 4: Frequency distribution of in-links per creator on their reference network.

Figure 4 depicts a frequency distribution of in-links per creator on each creation category. The distributions are mutually similar, but the highest number of in-link for Song Creation is lower and the curve is shallower than the others are, which indicates that the Song Creation Community is less centralized than others are.

\subsection{Community on Creators Network}

In this section, we analyze the creators' community. The term "creators' community" means a tight group of nodes within social network of creators. We adopt Newman clustering [5] to detect such communities from the social network of creators.

Newman clustering generated 83 clusters (communities) from the social network of creators. We especially investigated 10 clusters of which the size is greater than 50 .

We categorized these clusters into two types. Clusters 1 , 3,5 , and 6 have only one key person who has an extremely high number of in-links. On the other hand, clusters 2 and 4 do not clearly have a key person. Some creators have many more in-links than others in their community, but they are not dominant.

In each case, Songwriting is often a key person, meaning that Songwriting triggers creative activity. On the other hand, some communities have a key person from the Illustration category. These communities' development characterizes the Hatsune Miku and 3D modeling tool environment. Such communities have an important role in fostering creative activity.
Table 3: Structure of the biggest clusters. The columns of Key person and Majority respectively show categories of key persons and the majority.

\begin{tabular}{c|cccc}
\hline$\#$ & Size & Type & Key person & Majority \\
\hline 1 & 161 & centered & W & I \\
2 & 144 & messy & - & I \\
3 & 118 & centered & I\&C & I, C \\
4 & 95 & messy & - & I \\
5 & 91 & centered & I & I \\
6 & 90 & centered & W\&C & C \\
7 & 79 & centered & W & C \\
8 & 56 & messy & - & C,C C I \\
9 & 55 & centered & W\&C & C \\
10 & 51 & messy & - & I \\
\hline
\end{tabular}

\section{CONCLUSION}

As described in this paper, we have investigated how different types of creators interacted in massively collaborative creation. We extracted and analyzed the social networks of creators, revealing some interesting facts, e.g., different categories of creators have different roles in evolving the network.

Future TV is expected to be interactive, but interactivity means not merely that the audience becomes active in responding to TV programs even if they join to create content together. For that reason, what happens in video sharing sites is what can be expected to happen with future TV. The results presented in this paper are informative for future TV in this sense.

\section{ACKNOWLEDGMENTS}

This research was partially supported by KAKENHI.

\section{REFERENCES}

[1] C. Chen and R. J. Paul. Visualizing a knowledge domainĄf intellectual structure. Computer, 34(3):65-71, 2001.

[2] X. Cheng, C. Dale, and J. Liu. Statistics and social network of youtube videos. In Proc. of IWQoS2008, pages 229-238, 2008.

[3] M. J. Halvey and M. T. Keane. Exploring social dynamics in online media sharing. In Proc. of WWW2007, 2007.

[4] Y. Matsuo, J. Mori, M. Hamasaki, H. Takeda, T. Nishimura, K. Hashida, and M. Ishizuka. Polyphonet: An advanced social network extraction system. In Proc. of WWW2006, 2006.

[5] M. Newman. Fast algorithm for detecting community structure in networks. Phys. Rev. E, 69, 2004.

[6] H. Small. Co-citation in the scientific literature: A new measure of the relationship between two documents. Journal of the American Society of Information Science, 24:265-269, 1973. 